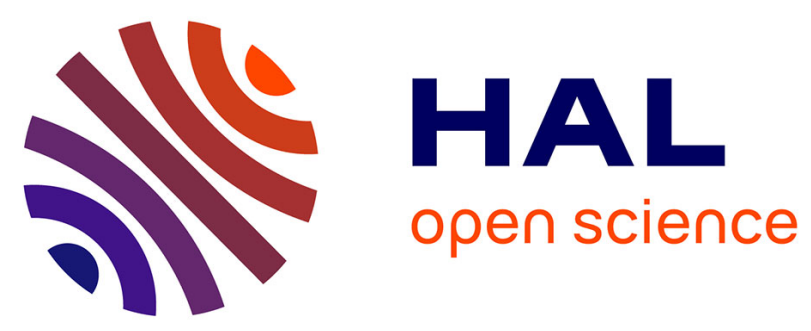

\title{
Numerical Solution of Time-Dependent Nonlinear Schrödinger Equations Using Domain Truncation Techniques Coupled With Relaxation Scheme
}

\author{
Xavier Antoine, Christophe Besse, Pauline Klein
}

\section{- To cite this version:}

Xavier Antoine, Christophe Besse, Pauline Klein. Numerical Solution of Time-Dependent Nonlinear Schrödinger Equations Using Domain Truncation Techniques Coupled With Relaxation Scheme. Laser Physics, 2011, 21 (8), pp.1-12. hal-00755612

\section{HAL Id: hal-00755612 \\ https://hal.science/hal-00755612}

Submitted on 21 Nov 2012

HAL is a multi-disciplinary open access archive for the deposit and dissemination of scientific research documents, whether they are published or not. The documents may come from teaching and research institutions in France or abroad, or from public or private research centers.
L'archive ouverte pluridisciplinaire HAL, est destinée au dépôt et à la diffusion de documents scientifiques de niveau recherche, publiés ou non, émanant des établissements d'enseignement et de recherche français ou étrangers, des laboratoires publics ou privés. 


\title{
Numerical solution of time-dependent nonlinear Schrödinger equations using domain truncation techniques coupled with relaxation scheme
}

\author{
X. Antoine $\mathrm{a}^{\mathrm{a}, 1, *}$, C. Besse $\mathrm{B}^{\mathrm{b}, 1}$, P. Klein ${ }^{\mathrm{a}, 1}$ \\ ${ }^{a}$ Institut Elie Cartan Nancy, Nancy-Université, CNRS UMR 7502, INRIA CORIDA Team, Boulevard des \\ Aiguillettes B.P. 239, 54506 Vandoeuvre-lès-Nancy, France \\ ${ }^{b}$ Laboratoire Paul Painlevé, Univ Lille Nord de France, CNRS UMR 8524, INRIA SIMPAF Team, \\ Université Lille 1 Sciences et Technologies, Cité Scientifique, 59655 Villeneuve d'Ascq Cedex, France
}

\begin{abstract}
The aim of this paper is to compare different ways for truncating unbounded domains for solving general nonlinear one- and two-dimensional Schrödinger equations. We propose to analyze Complex Absorbing Potentials, Perfectly Matched Layers and Absorbing Boundary Conditions. The time discretization is made by using a semi-implicit relaxation scheme which avoids any fixed point procedure. The spatial discretization involves finite element methods. We propose some numerical experiments to compare the approaches.
\end{abstract}

Keywords: Complex Absorbing Potentials, Absorbing Boundary Conditions, Perfectly Matched Layers, nonlinear Schrödinger equation, unbounded domain, relaxation scheme

\section{Introduction}

We propose and compare some numerical schemes to solve the general Schrödinger equations in unbounded media

$$
\left\{\begin{array}{l}
\left.i \partial_{t} \Psi^{\infty}(\mathbf{x}, t)+\Delta \Psi^{\infty}(\mathbf{x}, t)+V(\mathbf{x}, t) \Psi^{\infty}(\mathbf{x}, t)+f\left(\left|\Psi^{\infty}\right|\right) \Psi^{\infty}(\mathbf{x}, t)=0,(\mathbf{x}, t) \in \mathbb{R}^{N} \times\right] 0, T[ \\
\Psi^{\infty}(\mathbf{x}, 0)=\Psi_{0}(\mathbf{x}), \quad \mathbf{x} \in \mathbb{R}^{N} .
\end{array}\right.
$$

The wave function $\Psi^{\infty}$ is defined in the unbounded domain $\mathbb{R}^{N}(N \geq 1)$. In view of a numerical computation, different solutions may be used. For example, one usual scheme consists in splitting the Laplacian and potential-nonlinear parts of the equation and next solving the first linear equation e.g. by FFT methods and exactly integrating the second nonlinear one (see e.g. [1]). This kind of scheme is efficient and accurate if the solution remains confined within the computational domain (for instance for solving the Gross-Pitaevskii equations). Indeed, then periodic boundary conditions may be applied for the Fourier solution since

\footnotetext{
${ }^{*}$ Corresponding author.

Email addresses: Xavier.Antoine@iecn.u-nancy.fr (X. Antoine), Christophe.Besse@math.univ-lille1.fr (C. Besse), Pauline.Klein@iecn.u-nancy.fr (P. Klein) $U R L:$ http://www.iecn.u-nancy.fr/ antoine/ (X. Antoine), http://math.univ-lille1.fr/ besse/ (C. Besse)

${ }^{1}$ Supported partially by the French ANR fundings under the project MicroWave NT09_460489 (http://microwave.math.cnrs.fr/).
} 
the wave vanishes on the boundary of a large enough computational domain. In the same situation, other schemes may be used as for example Crank-Nicolson schemes, Runge-Kutta methods in time and finite difference or finite element in space. Spectral techniques may be applied too (see [2] for more details about some of these techniques). Independently of the numerical discretization, one common problem arises when the solution does not remain inside the computational domain. This is for example the case for the defocusing nonlinear cubic Schrödinger equation, for linear Schrödinger equation with laser ionization of a one-dimensional helium atom [3], for strong field laser atom interaction [4]... Then, it is well-known that Dirichlet or periodic boundary conditions on the boundary of the computational domain are not adapted. Our goal here is not to focus on all the physical situations which can arise but rather to propose some different ways of truncating accurately the computational domain for (1) and compare them numerically.

Concerning truncation methods, the case of the free Schrödinger equation is now mastered and many possible solutions can be developed. We refer to [5] for an overview of the techniques. Considering now the linear Schrödinger equation with potential requires more developments. For example, time dependent but space independent potentials can be considered easily by gauge change and be treated as the free-space case. The situation of a space variable potential is much more complex. In some exceptional cases, explicit exact boundary conditions may be written at the fictitious boundary. However, in most situations, approximate boundary conditions must be derived. These boundary conditions are usually called Absorbing Boundary Conditions (ABCs) since they try to absorb waves striking the nonphysical boundary. We refer to [5] for such examples in the one-dimensional case. In the two-dimensional case, only a few solutions can be found for the free- and potential cases [5]. In the nonlinear case which is much more complicate, the ABCs at hand are often formally built from the linear case with potential. To the best of our knowledge, only a few papers propose some ABCs $[6,7,8,9,10,11]$. For the two-dimensional case, the only strategies for simulating ABCs have been proposed in $[11,12]$. We propose and numerically test in this paper some new ABCs for (1) for the one- and two-dimensional cases which are related to the ABCs for potentials developed in $[13,14]$. A very common method in physics is related to Complex Absorbing Potentials (CAPs) [15]. The idea which is physically natural consists in adding to the linear Schrödinger equation a complex absorbing potential to damp the incoming wave in a surrounding layer. We try to extend this approach here to (1). As we will see, this approach fails to work and generates large reflections. In particular, the choice of the absorbing potential and its parameters is non trivial and extension to a nonlinear problem does not appear as natural. A related technique not analyzed here is the method of Exterior Complex Scaling (see examples in [16, 17]). A closely powerful method introduced by Bérenger [18] for electromagnetic waves is the Perfectly Matched Layer (PML) approach. The method introduces dissipation but inside the Laplacian term and not the potential term. We apply this technique here [19] to (1) to show its accuracy. It appears that the accuracy that can be expected from the ABCs and PMLs approaches is about the same, generally showing an error of reflection of the order of $0.1 \%$ or less, and is therefore useful for practical computations. For an easier implementation of all the truncation techniques, we use a semi-implicit relaxation scheme [20] which leads to a flexible implementation of the method. In particular, it does not require any iteration like in a fixed point or Newton procedure for the nonlinearity. Since the PML and ABCs approaches are accurate for the one-dimensional case, we next introduce their extension to two-dimensional problems. We detail the discretization issues and analyze their accuracy in the case of the propagation of 
a soliton in a cubic media.

The plan of the paper is the following. In Section 2, we introduce the ABCs, CAPs and PMLs techniques for the one-dimensional equation (1). We propose some schemes based on relaxation techniques coupled to Finite Element Methods (FEM). Finally, we numerically test and compare the different approaches. In the third Section, we extend our methods and discretizations to the two-dimensional nonlinear Schrödinger equation. Some numerical simulations confirm the accuracy of the methods. Finally, Section 4 gives a conclusion. Let us note here that the codes corresponding to Section 2 and 3 can be downloaded freely at http://microwave.math.cnrs.fr/code/index.html if the reader wants to know more about the implementation issues of all these techniques.

\section{One-dimensional nonlinear problems}

\subsection{Absorbing Boundary Conditions (ABCs)}

The first approach that we investigate concerns absorption at the boundary. We consider the time-dependent one-dimensional nonlinear Schrödinger equation with a variable potential and a nonlinear term

$$
\left\{\begin{array}{l}
i \partial_{t} \Psi(x, t)+\partial_{x}^{2} \Psi(x, t)+V(x, t) \Psi(x, t)+f(|\Psi|) \Psi(x, t)=0,(x, t) \in \Omega_{T}, \\
\Psi(x, 0)=\Psi_{0}(x), \quad x \in \Omega .
\end{array}\right.
$$

We assume here that $\Omega:=] x_{\ell}, x_{r}[$ represents a bounded computational domain of boundary $\Gamma:=\partial \Omega:=\left\{x_{\ell}, x_{r}\right\}$ and set $\left.\Omega_{T}:=\Omega \times\right] 0 ; T\left[, \Sigma_{t}:=\Sigma \times\right] 0 ; T[$ (see Figure 1).

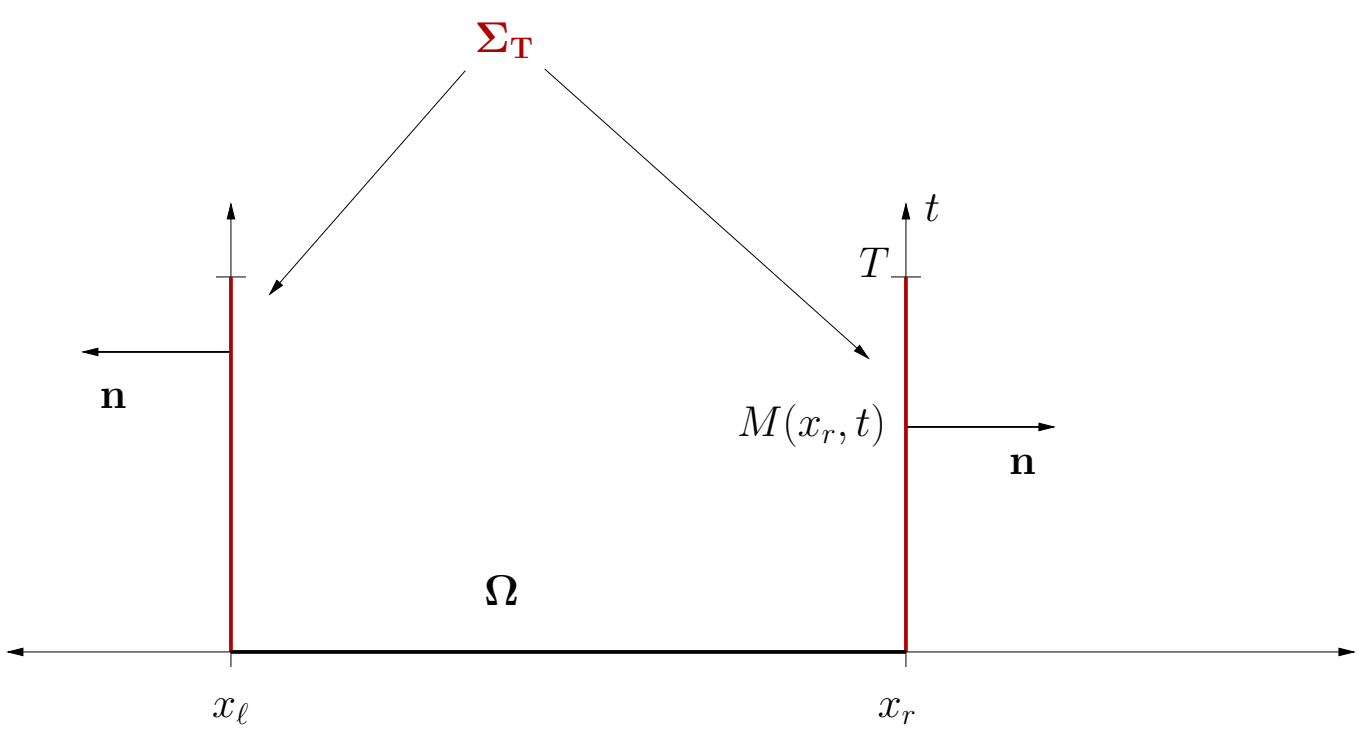

Figure 1: Computational domain $\Omega_{T}$ and fictitious boundary $\left.\Sigma_{T}=\Sigma \times\right] 0 ; T[$.

Furthermore, $\Psi_{0}$ is supposed to be a compactly supported initial data inside $\Omega$. If the potential $V$ and nonlinear interaction $f$ are constant outside $\Omega$, we respectively call them localized potential and interaction. Then exact absorption at the boundary $\Sigma$ can be obtained. To write this boundary condition (also called transparent), let us assume that the potentials are equal to zero outside $\Omega$. Then, it is now standard that the boundary condition is given by

$$
\partial_{\mathbf{n}} \Psi(x, t)+e^{-i \pi / 4} \partial_{t}^{1 / 2} \Psi(x, t)=0, \quad \text { on } \Sigma_{T},
$$


where $\mathbf{n}$ is the outwardly directed unit normal vector to $\Sigma$. The operator $\partial_{t}^{1 / 2}$ is the half-order derivative operator

$$
\partial_{t}^{1 / 2} \Psi(x, t):=\partial_{t} \int_{0}^{t} \frac{1}{\sqrt{\pi}} \frac{\Psi(x, \varrho)}{\sqrt{t-\varrho}} d \varrho
$$

It can be proved that system (2)-(3) is well-posed in a mathematical setting and that we have the energy bound

$$
\|\Psi(\cdot, t)\|_{0, \Omega} \leq\left\|\Psi_{0}(\cdot)\right\|_{0, \Omega},
$$

for any time $t>0$, where $\|u(\cdot, t)\|_{0, \Omega}$ designates the 2-norm of $\Psi$ over $\Omega$

$$
\|\Psi(\cdot, t)\|_{0, \Omega}=\left(\int_{\Omega}|\Psi(x, t)|^{2} d x\right)^{1 / 2}
$$

which can also be interpreted as the probability of finding $\Psi$ in $\Omega$ and translates the absorbing property of the boundary condition. The boundary condition (3) is exact in the sense that there is no reflection back into the computational domain. Mathematically, this implies that the solution to (2)-(3) is strictly equal to the restriction $\Psi_{\mid \Omega_{T}}^{\infty}$, solution to (1).

In the case of unbounded potential and interaction, then the situation is much more complex. Essentially, the possibility of writing the exact boundary condition (3) is related to the fact that for localized interactions, the Laplace transform can be used in the left and right exterior domains to write down the boundary condition through the Green function. In the case of nonlocal interactions, this no longer possible. Except in some special situations of potentials (e.g. linear potential) and nonlinearity (essentially integrable systems like the cubic case), it is impossible to get the exact expression of the absorption conditions. Here, we present without any mathematical details which are too cumbersome the boundary conditions that can be set at the boundary. We refer to [13] for the mathematical details. Essentially, the derivation is based on high-frequency asymptotic expansions in the Laplace domain using the extended theory of pseudodifferential operators. The resulting boundary conditions are no longer exactly non-reflecting. They are then called Absorbing (and not transparent) Boundary Conditions (ABCs), and we need to precise the order related to their asymptotics with the aim of measuring the a priori accuracy of the boundary condition. The $\mathrm{ABC}$ of order two is given by

$$
\partial_{\mathbf{n}} \Psi-i \sqrt{i \partial_{t}+V+f(|\Psi|)} \Psi=0,
$$

on $\Sigma_{T}$. The square-root operator of $i \partial_{t}+V+f(|\Psi|)$ means that we consider the spectral square-root decomposition of this operator. The resulting operator is nonlocal but will be localized later for the numerical purpose through Padé approximants. Higher-order ABCs can be derived [13] but will not be tested here. We cannot expect that an ABC works well for any potential. In practical computations and for $f=0$, one physically admissible assumption is that the potential is repulsive which means that $V: \mathbb{R} \times \mathbb{R}^{+}$is smooth and that we have $x \partial_{x} V(x, t)>0, \quad \forall(x, t) \in \Omega_{\ell, r} \times \mathbb{R}^{+}$, where $\left.\left.\Omega_{\ell}:=\right]-\infty ; x_{\ell}\right]$ and $\Omega_{r}:=\left[x_{r} ; \infty[\right.$. An example is $V(x, t)=\beta^{2}|x|^{a}$, with $0<a \leq 2$. The assumption on the nonlinearity is not clear most particularly when a potential is added. The only "intuitive" assumption is that the solution $\Psi$ is outgoing to the bounded domain and that no nonlinear or potential effect makes it reflecting back into $\Omega$, which is a priori difficult to check because mathematically hard to write. Finally, we can prove [13] that (5) still holds for the second-order ABC (6) and for a time independent potential $V(x)$ which translates the absorbing property of the boundary condition. 


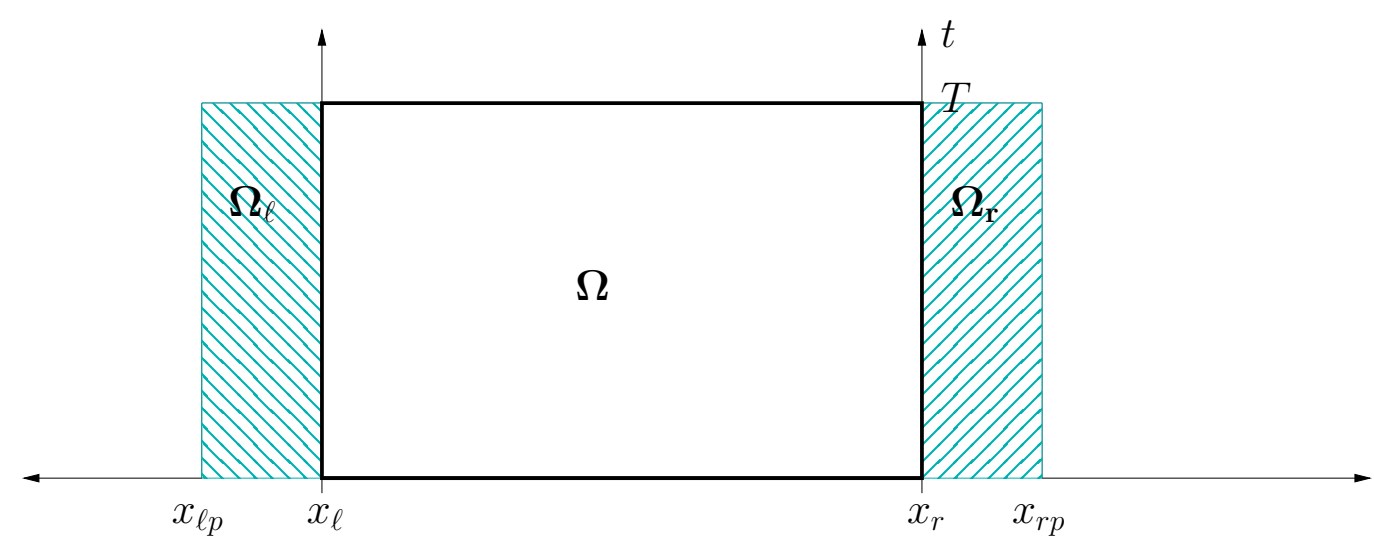

Figure 2: Computational domain for the CAP and PML approaches.

\subsection{Complex Absorbing Potential (CAP), Exterior Complex Scaling and Perfectly Matched Layers (PMLs)}

Another useful and widely studied approach for computing solutions to time-dependent Schrödinger equations with a potential term by using an absorbing domain is first the technique of Complex Absorbing Potential (CAP). Essentially, the idea consists in introducing a complex potential in the exterior domain to absorb the travelling wave. Mathematically, this consists in adding a spatial potential $-i W$ in some exterior layers $\left.\Omega_{\ell}=\right] x_{\ell p}, x_{\ell}[$ and $\left.\Omega_{r}=\right] x_{r}, x_{r p}$ [ (see Figure 2). Of course, to coincide with the solution to (2), $W$ is required to be zero in $\Omega$ and with a positive real part in the layers to damp the incoming wavefield. Another way to analyze this approach is called the Exterior Complex Scaling approach which consists in interpreting the introduction of the complex potential as the complex scaling: $x \rightarrow x e^{i \theta}$, where $\theta$ is a rotation angle which must be correctly chosen. Extension includes the Smooth Exterior Scaling approach $[16,17]$. From the numerical point of view, the CAP approach is direct to code since we have to solve

$$
\left\{\begin{array}{l}
i \partial_{t} \Psi(x, t)+\partial_{x}^{2} \Psi(x, t)+V(x, t) \Psi(x, t)-i W(x) \Psi(x, t)+f(|\Psi|) \Psi(x, t)=0,(x, t) \in \Omega_{T}^{e x t}, \\
\Psi(x, 0)=\Psi_{0}(x), \quad x \in \Omega^{e x t}
\end{array}\right.
$$

in the extended domain $\left.\Omega^{e x t}:=\Omega_{\ell} \cup \bar{\Omega} \cup \Omega_{r}=\right] x_{\ell p}, x_{r p}\left[\right.$ with boundary $\Sigma^{e x t}=\left\{x_{\ell p}, x_{r p}\right\}$. Here, according to $[16,21]$, we consider the quadratic profile

$$
W(x)= \begin{cases}W_{0} \delta^{-2}\left(x-x_{\ell}\right)^{2}, & x_{\ell p}<x<x_{\ell}, \\ 0, & x_{\ell}<x<x_{r}, \\ W_{0} \delta^{-2}\left(x-x_{r}\right)^{2}, & x_{r}<x<x_{r p}\end{cases}
$$

for a real positive value of $W_{0}$. The thickness $\delta$ of the layer is $\delta=\left|x_{r p}-x_{r}\right|=\left|x_{\ell p}-x_{\ell}\right|$. Other choices include exponential type absorbing functions [16]. At the boundary points $x_{\ell p}$ and $x_{r p}$, a boundary condition must be imposed. Here, we consider the classical homogeneous Dirichlet boundary conditions $\Psi(x, t)=0$ at $x_{\ell p}$ and $x_{r p}$. However, a suitable extension does not seem direct for nonlinear problems as we will see later.

We concentrate now on another closely related approach called the Perfectly Matched Layers (PMLs) method which was introduced by Bérenger [18] for Maxwell equations. The idea consists in introducing a complexification of the derivative operator through damping 
in the extended domains $\Omega_{\ell}$ and $\Omega_{r}$. In the case of the nonlinear Schrödinger equation, this can be written down as

$$
i \partial_{t} \Psi+\frac{1}{S(x)} \partial_{x}\left(\frac{1}{S(x)} \partial_{x} \Psi\right)+V(x, t) \Psi+f(|\Psi|) \Psi=0
$$

in $\Omega^{\text {ext }}$ (Figure 2). Function $S$ is given by $S(x):=1+R \sigma(x)$. The layer parameters $R$ and $\sigma$ must be chosen carefully. Optimization techniques and adaptive discretizations can be developed [5]. Here, we will use the parameter values derived in [19], i.e. $R=e^{i \pi / 4}$ and $\sigma$ is the quadratic function

$$
\sigma(x)= \begin{cases}\sigma_{0} \delta^{-2}\left(x-x_{\ell}\right)^{2}, & x_{\ell p}<x<x_{\ell} \\ 0, & x_{\ell}<x<x_{r} \\ \sigma_{0} \delta^{-2}\left(x-x_{r}\right)^{2}, & x_{r}<x<x_{r p}\end{cases}
$$

The distance $\delta:=\left|x_{r p}-x_{r}\right|=\left|x_{\ell}-x_{\ell p}\right|$ (that we take equal on both sides here for simplicity) corresponds to the thickness of the left and right absorption regions of the computational domain. Again, we fix the homogeneous Dirichlet boundary conditions: $\Psi(x, t)=0$ at $x_{\ell p}$ and $x_{r p}$. It is interesting to note the close form of CAP and PML approaches even if they lead to different equations to solve.

Unlike the ABCs, both CAP, ECS and PMLs must be adapted and optimized according to each situation. They have the advantage of being easy to code but at the price of an extended domain of computation $\Omega^{e x t}$ where the potential $V$ must be known. This is not always the case if $V$ is given only numerically for instance instead of analytically. One of the advantages of both CAP and PML methods is that they are local in time while it is not a priori the case for the ABCs which include a nonlocal square-root operator. However, this drawback can be removed by using local approximations as seen later. Finally, all the techniques are derived in the linear situation and their application to nonlinear problems is formal. Therefore, their accuracy must be prospected.

\subsection{Discretization schemes}

To compute the solution of the previous problems, we have to introduce some numerical discretizations. First, we have to deal with the time discretization in the interior domain. One widely used scheme is the Crank-Nicolson scheme which reads

$$
2 i \frac{\Phi^{n+1}}{\Delta t}+\partial_{x}^{2} \Phi^{n+1}+W^{n+1} \Phi^{n+1}+\left(\frac{f\left(\left|\Psi^{n+1}\right|\right)+f\left(\left|\Psi^{n}\right|\right)}{2}\right) \Phi^{n+1}=2 i \frac{\Psi^{n}}{\Delta t}
$$

for $0 \leq n \leq N-1$, and a time step $\Delta t=T / N$. Here, we set

$$
W^{n+1}=\frac{V^{n+1}+V^{n}}{2}, \Phi^{n+1}=\frac{\Psi^{n+1}+\Psi^{n}}{2} .
$$

A more adapted scheme to the computation of soliton solutions is the Duran-Sanz-Serna scheme [22]. Unfortunately, in both cases, the scheme remains nonlinear and a fixed point or a Newton algorithm is required. A few iterations are then necessary increasing the global computational cost of the procedure. Instead of using these numerical methods, we can 
consider the Besse relaxation scheme in [20]. Applied to the nonlinear equation $i \partial_{t} \Psi+$ $\partial_{x}^{2} \Psi+V \Psi+f(|\Psi|) \Psi=0$, on $\Omega_{T}$, the method leads to the solution to

$$
\left\{\begin{array}{l}
i \partial_{t} \Psi+\partial_{x}^{2} \Psi+V \Psi+\Upsilon \Psi=0, \quad \text { on } \Omega_{T} \\
\Upsilon=f(|\Psi|), \quad \text { on } \Omega_{T}
\end{array}\right.
$$

Then, system (13) is discretized as

$$
\left\{\begin{array}{l}
2 i \frac{\Phi^{n+1}}{\Delta t}+\partial_{x}^{2} \Phi^{n+1}+W^{n+1} \Phi^{n+1}+\Upsilon^{n+1 / 2} \Phi^{n+1}=2 i \frac{\Psi^{n}}{\Delta t}, \\
\frac{\Upsilon^{n+3 / 2}+\Upsilon^{n+1 / 2}}{2}=f\left(\left|\Psi^{n+1}\right|\right),
\end{array}\right.
$$

for $0 \leq n \leq N-1$. The initialization of $\Upsilon$ is chosen as $\Upsilon^{-1 / 2}=f\left(\left|\Psi^{0}\right|\right)$. We can see that this time discretization avoid any additional iterative procedure for the nonlinear term and so is well adapted to nonlinear problems. Furthemore, this scheme is known to preserve many invariants for the nonlinear Schrödinger equation [20].

In the case of the CAP, ECS and PML techniques, the relaxation scheme directly applies on $\Omega^{e x t}$. In the ABCs case, we have to discretize correctly the square-root operator. To this aim, we can approximate this operator by using Padé approximants $R_{m}$ and $\Upsilon$

$$
\partial_{\mathbf{n}} \Psi-i R_{m}\left(i \partial_{t}+V+\Upsilon\right) \Psi=0 .
$$

The function $R_{m}$ is defined by

$$
R_{m}(z)=a_{0}^{m}+\sum_{k=1}^{m} \frac{a_{k}^{m} z}{z+d_{k}^{m}}=\sum_{k=0}^{m} a_{k}^{m}-\sum_{k=1}^{m} \frac{a_{k}^{m} d_{k}^{m}}{z+d_{k}^{m}}
$$

where the coefficients $a_{k}^{m}$ and $d_{k}^{m}$ are given by $a_{0}^{m}=1$, and for $1 \leq k \leq m$

$$
a_{k}^{m}=\frac{1}{m \cos ^{2}\left(\frac{(2 k-1) \pi}{4 m}\right)}, \quad d_{k}^{m}=\tan ^{2}\left(\frac{(2 k-1) \pi}{4 m}\right) .
$$

We can explicit this relation (formally with $z=i \partial_{t}+V+\Upsilon$ ) by

$$
\partial_{\mathbf{n}} \Psi-i\left(\sum_{k=0}^{m} a_{k}^{m} \Psi-\sum_{k=1}^{m} a_{k}^{m} d_{k}^{m}\left(i \partial_{t}+V+\Upsilon+d_{k}^{m}\right)^{-1} \Psi\right)=0 .
$$

Then we introduce some auxiliary functions $\left(\varphi_{k}\right)_{1 \leq k \leq m}$ defined by

$$
\varphi_{k}=\left(i \partial_{t}+V+\Upsilon+d_{k}^{m}\right)^{-1} \Psi
$$

for the square-root approximation. The ABC then becomes

$$
\partial_{\mathbf{n}} \Psi-i \sum_{k=0}^{m} a_{k}^{m} \Psi+i \sum_{k=1}^{m} a_{k}^{m} d_{k}^{m} \varphi_{k}=0
$$

and the time discretization is

$$
\partial_{\mathbf{n}} \Phi^{n+1}-i \sum_{k=0}^{m} a_{k}^{m} \Phi^{n+1}+i \sum_{k=1}^{m} a_{k}^{m} d_{k}^{m} \varphi_{k}^{n+1 / 2}=0 .
$$


The definitions of the auxiliary functions lead to the coupled differential equations

$$
i \partial_{t} \varphi_{k}+(V+V+\Upsilon) \varphi_{k}+d_{k}^{m} \varphi_{k}=\Psi, \quad x=x_{\ell, r}
$$

These are discretized by $\varphi_{k}^{n+1 / 2}=\frac{\varphi_{k}^{n+1}+\varphi_{k}^{n}}{2}$ with

$$
\frac{2 i}{\Delta t} \varphi_{k}^{n+1 / 2}+\left(W^{n+1}+\Upsilon^{n+1 / 2}\right) \varphi_{k}^{n+1 / 2}+d_{k}^{m} \varphi_{k}^{n+1 / 2}=\Phi^{n+1}+\frac{2 i}{\Delta t} \varphi_{k}^{n}, \quad x=x_{\ell, r} .
$$

This gives an explicit expression for the auxiliary functions at $x=x_{\ell, r}$

$$
\varphi_{k}^{n+1 / 2}=\frac{1}{\frac{2 i}{\Delta t}+W^{n+1}+\Upsilon^{n+1 / 2}+d_{k}^{m}} \Phi^{n+1}+\frac{\frac{2 i}{\Delta t}}{\frac{2 i}{\Delta t}+W^{n+1}+\Upsilon^{n+1 / 2}+d_{k}^{m}} \varphi_{k}^{n}
$$

Injecting (19) into (18), we get an explicit expression of $\partial_{\mathbf{n}} \Psi^{n+1}$ in terms of $\Psi^{n+1}$ and other updated functions. To discretize the problem in space, we use the weak formulation associated to (14) which writes down for any test function $\varphi$,

$$
\begin{aligned}
& \frac{2 i}{\Delta t} \int_{\Omega} \Phi^{n+1} \varphi d \Omega-\int_{\Omega} \partial_{x} \Phi^{n+1} \partial_{x} \varphi d \Omega+\int_{\Sigma} \partial_{\mathbf{n}} \Phi^{n+1} \varphi d \Sigma \\
& \quad+\int_{\Omega}\left(W^{n+1 / 2}+\Upsilon^{n+1 / 2}\right) \Phi^{n+1} \varphi d \Omega=\frac{2 i}{\Delta t} \int_{\Omega} \Psi^{n} \varphi d \Omega
\end{aligned}
$$

with the update of $\Upsilon$ on $\Omega$

$$
\Upsilon^{n+3 / 2}=2 f\left(\left|\Phi^{n+1}\right|\right)-\Upsilon^{n+1 / 2}
$$

We can see here that the nonlinearity is just included as a potential and therefore no fixed point or Newton method is necessary since the nonlinearity is explicit through the update (21). This is also definitively the way the boundary condition (18) on $\Sigma$ is treated. The ABCs are then just a Fourier-Robin boundary condition which can be easily implemented in the finite element code. The formulation (20) can then be solved through (high-order) finite element methods, resulting in the solution of a linear system at each time step. In the case of a CAP, ECS or PML, the adaptation is direct by integrating (13) over $\Omega^{\text {ext }}$ and

by using the homogeneous Dirichlet boundary condition. In the sequel, we use linear finite element methods. The domain is decomposed into $n_{h}$ spaced elementary segments of size $h$. Other spatial discretization methods could be used such as the finite difference or spectral techniques.

\subsection{Numerical examples}

\subsubsection{Soliton propagation in a cubic media}

The first test case concerns the propagation of a soliton in a cubic nonlinear media $(V=0$ and $\left.f(|\Psi|)=|\Psi|^{2}\right)$

$$
\Psi^{\text {exact }}(x, t)=2 \operatorname{sech}\left(\sqrt{2}\left(x-k_{0} t\right)\right) \exp \left(i \frac{k_{0}}{2}\left(x-k_{0} t\right)+i\left(2+k_{0}^{2} / 4\right) t\right)
$$

for the wavenumber $k_{0}=15$. The computational domain is $[-10 ; 10]$ and the maximal time of computation is $T=2$. We take the time step $\Delta t=10^{-3}$ and $n_{h}=2000$ points for the uniform spatial discretization. For the CAP, the size of the layer is $\delta=4$ and $W_{0}=10$ for 
$n_{h}=2800$. The same domain is considered for the PML with $\sigma_{0}=10$. For the ABC, we take $m=50$ Padé functions. We represent the amplitude of $\Psi$ in normal but also logarithmic scale to observe the small reflections which may appear during the numerical solution and to understand the accuracy improvement. We clearly see on Figures 3(a)-3(b) that the CAP approach gives good results until a wave comes back into the computational domain. On figures 3(c)-3(f), the PML and ABC methods yields some good results with about the same accuracy. Low amplitude waves are reflected back into the computational domain (about $0.1 \%$ of the maximal amplitude of the soliton). For the PML, the size of the computational domain is $40 \%$ more than for the ABCs. Finally, a very good accuracy is obtained for the relaxation scheme which makes it very attractive.

\subsubsection{Numerical simulation of a saturable nonlinear Schrödinger equation}

The second test that we present concerns the numerical solution of a saturable Schrödinger equation (SNLSE) used in optics. This equation models pulse propagation in optical fibers made from doped silica [23]. The saturating nonlinear term is exponential and the equation that we solve is

$$
\left\{\begin{array}{l}
i \partial_{t} \Psi(x, t)+\partial_{x}^{2} \Psi(x, t)+\frac{|\Psi|^{2}}{1+\gamma|\Psi|^{2}} \Psi(x, t)=0,(x, t) \in \Omega_{T}, \\
\Psi(x, 0)=\Psi_{0}(x), \quad x \in \Omega
\end{array}\right.
$$

The saturation term $\gamma$ is related to the saturation intensity of the fiber. We take $\gamma=0.5$ to be close to the values in [23]. The initial datum is the soliton $(22)$ (for $t=0$ ). All the simulations parameters are the same as in Section 2.4.1. We can again see that the CAP method leads to wrong results while the PML and ABC approaches provide a good accuracy for a small reflection. The relaxation scheme is efficient and accurate.

\section{Two-dimensional nonlinear problems}

Let us consider the two-dimensional nonlinear Schrödinger equation

$$
\left\{\begin{array}{l}
i \partial_{t} \Psi(\mathbf{x}, t)+\Delta \Psi(\mathbf{x}, t)+V(\mathbf{x}, t) \Psi(\mathbf{x}, t)+f(|\Psi|) \Psi(\mathbf{x}, t)=0,(\mathbf{x}, t) \in \Omega_{T}, \\
\Psi(\mathbf{x}, 0)=\Psi_{0}(\mathbf{x}), \quad \mathbf{x} \in \Omega .
\end{array}\right.
$$

Here, the computational domain $\Omega$ is a bounded set of $\mathbb{R}^{2}$ with a regular convex boundary $\Sigma$. We compute the solution on a time interval $[0 ; T]$. The resulting time-space domains are therefore: $\Omega_{T}:=\Omega \times[0 ; T]$, and $\Sigma_{T}:=\Sigma \times[0 ; T]$. The initial data $\Psi_{0}$ is compactly supported in $\Omega$. The operator $\Delta$ is the Laplace operator in two-dimensions: $\Delta:=\partial_{x_{1}}^{2}+\partial_{x_{2}}^{2}$, with $\mathbf{x}=\left(x_{1}, x_{2}\right)$. The potential $V$ is a function from $\mathbb{R}^{2} \times[0 ; T]$ onto $\mathbb{R}$ which is smooth outside $\Omega$. The nonlinear function $f$ is supposed to be smooth outside $\Omega$.

\subsection{Absorbing Boundary Conditions}

The development of ABCs for two-dimensional nonlinear Schrödinger equation is a very recent area of research. Very few results are only available. We refer to $[11,12]$ for examples. In the present paper, we consider the new ABCs developed in $[13,14]$. Again, we do not explain the technical theory for constructing such boundary conditions and refer to [13, 14] for more details. The ABC for the two-dimensional case is

$$
\begin{aligned}
\partial_{\mathbf{n}} \Psi- & i \sqrt{i \partial_{t}+\Delta_{\Sigma}+V+f(|\Psi|)} \Psi+\frac{\kappa}{2} \Psi \\
& -\frac{\kappa}{2}\left(i \partial_{t}+\Delta_{\Sigma}+V+f(|\Psi|)\right)^{-1} \Delta_{\Sigma} \Psi=0, \quad \text { on } \Sigma_{T} .
\end{aligned}
$$


This boundary condition can be considered as an extension of (6). Vector $\mathbf{n}$ is the outwardly directed unit normal vector to $\Omega$. The function $\kappa$ is the curvature of $\Sigma$ at a point of the surface. Finally, $\Delta_{\Sigma}$ is the Laplace-Beltrami operator over the surface. For example, for a disk of radius $R$, the normal derivative is $\partial_{\mathbf{n}}=\partial_{r}$, the curvature is $R^{-1}$ and $\Delta_{\Sigma}=R^{-2} \partial_{\theta}^{2}$, where the polar coordinate system is $(r, \theta)$. We will show during the numerical approximation by the relaxation scheme how to suitably localize this operator.

\subsection{Complex Absorbing Potential and Perfectly Matched Layers}

The extension of the CAP method is direct in the form

$$
\left\{\begin{array}{l}
i \partial_{t} \Psi(\mathbf{x}, t)+\Delta \Psi(\mathbf{x}, t)+V(\mathbf{x}, t) \Psi(\mathbf{x}, t)-i W(\mathbf{x}) \Psi(\mathbf{x}, t)+f(|\Psi|) \Psi(\mathbf{x}, t)=0,(\mathbf{x}, t) \in \Omega_{T}^{e x t} \\
\Psi(\mathbf{x}, 0)=\Psi_{0}(\mathbf{x}), \quad \mathbf{x} \in \Omega^{e x t}
\end{array}\right.
$$

The choice of the function $W$ is however not clear most particularly for a nonlinear problem and a general domain $\Omega$. Here, the domain $\Omega$ is the rectangle $]-a_{1}, a_{1}[\times]-a_{2}, a_{2}[$, and the extended computational domain $\Omega^{e x t}$ is $]-\left(a_{1}+\delta\right), a_{1}+\delta[\times]-\left(a_{2}+\delta\right), a_{2}+\delta[$. The thickness of the layer is parameterized by $\delta$. We use $W(\mathbf{x})=W_{1}(\mathbf{x}) W_{2}(\mathbf{x})$, where $W_{j}$ is given by

$$
W_{j}(\mathbf{x})=W_{0} \delta^{-2}\left(\left|x_{j}\right|-a_{j}\right)^{2} \mathbf{1}_{\left|x_{j}\right| \geq a_{j}}(\mathbf{x}) .
$$

Function $\mathbf{1}_{\mathcal{O}}$ is the characteristic function of a set $\mathcal{O}$. It is not clear if this choice is optimal. We do not develop the ECS here.

For the PMLs approach, the choice of domain $\Omega^{e x t}$ is restricted in practice since essentially the PMLs are written according to a special coordinates system related to $\Omega$. For example, for the cartesian coordinates associated with the rectangles $\Omega$ and $\Omega^{e x t}$, we consider the modified system

$$
\left\{\begin{array}{l}
i \partial_{t} \Psi+\frac{1}{S_{1} S_{2}}\left(\partial_{x_{1}}\left(\frac{S_{1}}{S_{2}} \partial_{x_{1}} \Psi\right)+\partial_{x_{2}}\left(\frac{S_{2}}{S_{1}} \partial_{x_{2}} \Psi\right)\right)+V \Psi+f(|\Psi|) \Psi=0,(\mathbf{x}, t) \in \Omega_{T}^{e x t} \\
\Psi(\mathbf{x}, 0)=\Psi_{0}(\mathbf{x}), \quad \mathbf{x} \in \Omega \\
\Psi(\mathbf{x}, t)=0, \quad \mathbf{x} \in \Sigma_{T}^{e x t}
\end{array}\right.
$$

with, for $j=1,2$,

$$
\left\{\begin{array}{l}
S_{j}(\mathbf{x})=1+R \sigma_{j}(\mathbf{x}) \\
\sigma_{j}(\mathbf{x})=\sigma_{0}\left(\frac{\left|x_{j}\right|-a_{j}}{\delta}\right)^{2} \mathbf{1}_{\left|x_{j}\right| \geq a_{j}}(\mathbf{x})
\end{array}\right.
$$

Let us now consider an annulus. The physical computational domain is the disk of radius $\Omega=D_{R}$ and the PML medium is the annulus $D_{\delta}$ of thickness $\delta=R^{*}-R$. Then, going to the polar coordinates system $(r, \theta)$, the PML writes down

$$
\left\{\begin{array}{l}
i \partial_{t} \Psi+\frac{1}{r \hat{S} S}\left[\partial_{r}\left(\frac{\hat{S} r}{S} \partial_{r} \Psi\right)+\frac{S}{\hat{S} r} \partial_{\theta}^{2} \Psi\right]+V \Psi+f(|\Psi|) \Psi=0,(\mathbf{x}, t) \in \Omega_{T}^{e x t} \\
\Psi(\mathbf{x}, 0)=\Psi_{0}(\mathbf{x}), \quad \mathbf{x} \in \Omega \\
\Psi(\mathbf{x}, t)=0, \quad \mathbf{x} \in \Sigma_{T}^{e x t}
\end{array}\right.
$$


with $\Omega^{e x t}=D_{R^{*}}, \Sigma^{e x t}=C_{R^{*}}$ and

$$
\left\{\begin{array}{l}
S(r)=1+R \sigma(r) \\
\hat{S}(r)=1+\frac{R}{r} \int_{R}^{r} \sigma(s) d s \\
\sigma(r)=\sigma_{0}\left(\frac{r-R}{\delta}\right)^{2} \mathbf{1}_{r \geq R}(\mathbf{x})
\end{array}\right.
$$

\subsection{Discretization schemes}

For the Equation (24), the interior relaxation scheme which is based on

$$
\left\{\begin{array}{l}
i \partial_{t} \Psi+\Delta \Psi+V \Psi+\Upsilon \Psi=0, \quad \text { on } \Omega_{T} \\
\Upsilon=f(|\Psi|), \quad \text { on } \Omega_{T}
\end{array}\right.
$$

is given by

$$
\left\{\begin{array}{l}
2 i \frac{\Phi^{n+1}}{\Delta t}+\Delta \Phi^{n+1}+W^{n+1} \Phi^{n+1}+\Upsilon^{n+1 / 2} \Phi^{n+1}=2 i \frac{\Psi^{n}}{\Delta t} \\
\frac{\Upsilon^{n+3 / 2}+\Upsilon^{n+1 / 2}}{2}=f\left(\left|\Psi^{n+1}\right|\right)
\end{array}\right.
$$

for $0 \leq n \leq N-1$, where the notations are the same as (12).

In the case of the $\mathrm{ABC}(25)$, we discretize the relation by using $\Upsilon=f(|\Psi|)$ and some additional auxiliary functions $\left(\varphi_{k}\right)_{1 \leq k \leq m}$ and $\psi$. More precisely, we get the scheme

$$
\left\{\begin{array}{l}
\partial_{\mathbf{n}} \Phi^{n+1}-i\left(\sum_{k=0}^{m} a_{k}^{m}\right) \Phi^{n+1}+\frac{\kappa}{2} \Phi^{n+1}+i \sum_{k=1}^{m} a_{k}^{m} d_{k}^{m} \varphi_{k}^{n+1 / 2}-\frac{\kappa}{2} \psi^{n+1 / 2}=0, \quad \text { on } \Sigma, \\
\left(\frac{2 i}{\Delta t}+\Delta_{\Sigma}+W^{n+1}+\Upsilon^{n+1 / 2}+d_{k}^{m}\right) \varphi_{k}^{n+1 / 2}-\Phi^{n+1}=\frac{2 i}{\Delta t} \varphi_{k}^{n}, \quad \text { on } \Sigma, \\
\left(\frac{2 i}{\Delta t}+\Delta_{\Sigma}+W^{n+1}+\Upsilon^{n+1 / 2}\right) \psi^{n+1 / 2}-\Delta_{\Sigma} \Phi^{n+1}=\frac{2 i}{\Delta t} \psi^{n}, \quad \text { on } \Sigma, \\
\varphi_{k}^{0}=0 \quad \text { on } 1 \leq k \leq m, \quad \psi^{0}=0, \quad \text { on } \Sigma \\
\Upsilon^{n+3 / 2}=2 f\left(\left|\Phi^{n+1}\right|\right)-\Upsilon^{n+1 / 2}, \quad \text { on } \Omega .
\end{array}\right.
$$

In view of a finite element approach, we need to write the associated weak formulation for the first equation of system (32), for a test-function $\varphi$,

$$
\begin{gathered}
\frac{2 i}{\Delta t} \int_{\Omega} \Phi^{n+1} \varphi d \Omega-\int_{\Omega} \nabla \Phi^{n+1} \cdot \nabla \varphi d \Omega+\int_{\Sigma} \partial_{\mathbf{n}} \Phi^{n+1} \varphi d \Sigma+\int_{\Omega} W^{n+1} \Phi^{n+1} \varphi d \Omega \\
+\int_{\Omega} \Upsilon^{n+1 / 2} \Phi^{n+1} \varphi d \Omega=\frac{2 i}{\Delta t} \int_{\Omega} \Psi^{n} \varphi d \Omega
\end{gathered}
$$

and updating by

$$
\Upsilon^{n+3 / 2}=2 f\left(\left|\Psi^{n+1}\right|\right)-\Upsilon^{n+1 / 2}
$$

Then, we inject the expression of the normal derivative $\partial_{\mathbf{n}} \Phi^{n+1}$ by using (33). This leads to a linear system of equations with unknowns $\Phi^{n+1}$ in $\Omega$, coupled to the surface equations in terms of additional unknowns $\left(\varphi_{k}\right)_{1 \leq k \leq m}$ and $\psi$. For the CAP and PML, both the time and space discretizations are directly realized in the extended computational domain. Finite difference are used for the spatial discretization. 


\subsection{The example of the cubic media for the propagation of a soliton}

\subsubsection{Soliton construction by a shooting method}

In the two-dimensional case, there is no explicit analytical expression of the soliton. Its construction must be realized numerically. To this aim, we compute a solution to the 2D stationary Schrödinger equation by using a shooting method [24]. Let us consider the nonlinear focusing cubic Schrödinger equation

$$
i \partial_{t} \psi+\Delta \psi+|\psi|^{2} \psi=0, \quad \text { on } \mathbb{R}^{2} \times \mathbb{R}^{+},
$$

and let us compute a stationary solution under the form

$$
\psi(r, t)=e^{i \mu t} \phi(r),
$$

where $r=\|\mathbf{x}\|=\sqrt{x^{2}+y^{2}}, \mu \in \mathbb{R}$ and $\phi$ is supposed to be spatially localized. Then we have to solve the nonlinear elliptic equation

$$
-\mu \phi+\Delta \phi+|\phi|^{2} \phi=0, \quad \mathbf{x} \in \mathbb{R}^{2} .
$$

We make the assumption that $\phi$ has a radial symmetry. Then going to the radial coordinates, we have to solve a second-order ordinary differential equation on the interval $[0 ; R]$

$$
\partial_{r}^{2} \phi+\frac{1}{r} \partial_{r} \phi-\mu \phi+|\phi|^{2} \phi=0, \quad r \in[0 ; R] .
$$

We can next work for $\mu=1$ by the change of variable

$$
\tilde{\phi}(r)=\frac{1}{\sqrt{\mu}} \phi\left(\frac{r}{\sqrt{\mu}}\right) .
$$

Furthermore, to get a two differentiable smooth solution we impose $\psi^{\prime}(0)=0$ to avoid any singularity at 0 . Finally, we have to solve the differential nonlinear system

$$
\left\{\begin{array}{l}
\partial_{r}^{2} \phi+\frac{1}{r} \partial_{r} \phi-\phi+|\phi|^{2} \phi=0, \quad 0<r<R, \\
\phi^{\prime}(0)=0, \quad \phi(0)=\beta,
\end{array}\right.
$$

where we try to find a solution which tends towards zero to infinity. A Taylor expansion at zero shows that we must have $\phi^{\prime \prime}(0)=\beta-q|\beta|^{2} \beta$. A shooting method is then applied by taking a radial step of discretization $\Delta r=10^{-3}$. The initial data $\beta$ is ajusted in such a way that suitable decay of the solution $\phi$ is obtained. We therefore have a radial solution $\phi(r)$, for $r \leq R$, which is then extended to the whole disk of radius $R$ by radial symmetry to get the soliton solution. Then, the soliton is multiplied by a gaussian with wavenumber $\mathbf{k}_{0}=k_{0} x$ as

$$
\psi_{0}(x, y)=\phi(r) e^{-i k_{0} x_{1}}
$$

to make it moves. At $R=10$, we have the approximation $\left|\psi_{0}(R)\right| \approx 5 \times 10^{-5}$. If we extend the domain to $R=15$, we get $\left|\psi_{0}(R)\right| \approx 3 \times 10^{-7}$. Indeed, the soliton has a slow decay which implies that relatively large computational domains must be chosen to be sure that the initial data is numerically compactly supported. At the computer code level, this implies that we have to work in quadruple precision for $R=15$. For stability reasons, the number of significative digits is crucial for $\beta$. Finally, we will consider next the disk of radius $R=10$ as computational domain $\Omega$. 
Remark 1. This construction of the soliton directly extends to other nonlinearities such as $f(|\psi|)=|\psi|^{2 \sigma}$, with $\sigma>0$. This allows for example to consider the quintic nonlinearity $f(|\psi|)=|\psi|^{4}$. Let us remark that other possible applications of interest of the shooting techniques (for stationary solutions) as well as relaxation schemes (for nonlinear dynamics) could be for instance the numerical solution of nonlinear Schrödinger equations modelling DC and AC Josephson effects for superfluid Fermi in BCS-BEC crossover at zero temperature $[25,26]$. Then, specific potentials must be considered for realistic simulations. In the same spirit, applications could be considered in the background of Feshback resonance where specific nonlinear potentials with trapping potential and external driving field are included [27].

\subsubsection{Accuracy of the truncation techniques}

We now consider as initial data the soliton solution computed by the previous shooting method and then modulated by $e^{i k_{0} x_{1}}$ with $k_{0}=5$. For the CAP and PMLs, we take $\Delta t=10^{-3}$ for a rectangular physical domain $\Omega:=[-10,10] \times[-10,10]$ embedded in the extended domain $\Omega^{e x t}:=[-12,12] \times[-12,12]$. This last domain is discretized with a uniform grid composed of $501 \times 501$ points for the finite difference approximation. For the ABC approach, the domain is the circle of radius 10 for $n_{P}=220000$ degrees of freedom of the linear finite element method. Figure 5(a) represents the 3D propagation of the soliton solution $|\psi|$ in the space $\left(x_{1}, x_{2}, t\right)$ with $\mathrm{ABCs}$ in log-scale. We can observe some small reflections at the boundary when the soliton hits the left plane. For an easier visualization of the results, we choose to report a slice of the wave field in the plane $\left(x_{1}, t\right)$ for $x_{2}=0$ again in log-scale. This gives Figures 5(b)-5(d) for respectively the CAPs, PMLs and ABCs approaches. As in the one-dimensional case, the proposed CAP method gives incorrect results while both PMLs and ABCs solution are physically correct with small reflection back. Furthermore, the relaxation schemes yield again a suitable accuracy for a low computational cost.

\section{Conclusion}

We proposed in this paper a numerical comparison of CAPs, PMLs and ABCs techniques for one- and two-dimensional nonlinear Schrödinger equations (cubic and saturation media). The initial data is a soliton which is analytically known for the one-dimensional case and numerically built by a shooting method in the two-dimensional case. The numerical schemes are based on a relaxation scheme in time and finite element or finite difference in space. From the numerical simulations, it appears that the PMLs and ABCs provide a suitable and similar accuracy compared to the CAPs method which leads to wrong results. Finally, all the computer codes are available freely at http://microwave.math.cnrs.fr/code/index.html.

[1] W. Bao, D. Jaksch, P. Markowich, J. Comput. Phys. 187318 (2003).

[2] W. Bao, The nonlinear Schrödinger equation and applications in Bose-Einstein condensation and plasma physics, in: Dynamics in Models of Coarsening, Condensation and Quantization, Vol. 9 of IMS Lecture Notes Series, (World Scientific 2007), p. 215.

[3] S. Selsto, S. Kvaal, J. Phys. B-At. Mol. Opt. Phys. 43065004 (2010).

[4] M. Heinen, H. J. Kull, Laser Phys. 20581 (2010). 
[5] X. Antoine, A. Arnold, C. Besse, M. Ehrhardt, A. Schädle, Comm. Comput. Phys. 4 729 (2008).

[6] X. Antoine, C. Besse, S. Descombes, SIAM J. Numer. Anal. 432272 (2006).

[7] J. Szeftel, Numer. Math. 104103 (2006).

[8] X. Antoine, C. Besse, J. Szeftel, Cubo 1129 (2009).

[9] C. Zheng, J. Comput. Phys. 215552 (2006).

[10] J. Zhang, Z. Xu, X. Wu, Phys. Rev. E 78026709 (2008).

[11] Z. Xu, H. Han, X. Wu, J. Comput. Phys. 2251577 (2007).

[12] J. Zhang, Z. Xu, X. Wu, Phys. Rev. E 79046711 (2009).

[13] P. Klein, Construction et analyse de conditions aux limites artificielles pour des équations de Schrödinger avec potentiels et non linéarités, Ph.D. thesis, Nancy Université, France (November, 2010).

[14] X. Antoine, C. Besse, P. Klein, in preparation.

[15] J. Muga, J. Palao, B. Navarro, I. Egusquiza, Phys. Rep.-Rev. Sec. Phys. Lett. 395357 (2004).

[16] A. Scrinzi, Phys. Rev. A 81053845 (2010).

[17] O. Shemer, D. Brisker, N. Moiseyev, Phys. Rev. A 71032716 (2005).

[18] J. Bérenger, J. Comput. Phys. 114185 (1994).

[19] C. Zheng, J. Comput. Phys. 227537 (2007).

[20] C. Besse, SIAM J. Numer. Anal. 42934 (2004).

[21] A. Jungel, J.-F. Mennemann, Math. Comput. Simul. In Press.

[22] A. Durán, J. M. Sanz-Serna, IMA J. Numer. Anal. 20235 (2000).

[23] A. Usman, J. Osman, D. Tilley, Turkish Journal of Physics 2817 (2004).

[24] L. Di Menza, Math. Mod. Num. Anal. 43173 (2009).

[25] L. Salasnich, Laser Phys. 19642 (2009).

[26] L. Salasnich, F. Ancilotto, N. Manini, F. Toigo, Laser Phys. 19636 (2009).

[27] V. Yukalov, V. Bagnato, Laser Phys. Lett. 6399 (2009). 


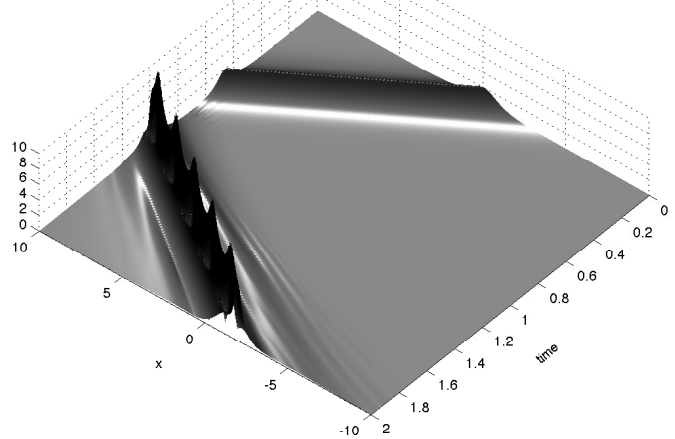

(a) $\mathrm{CAP}$

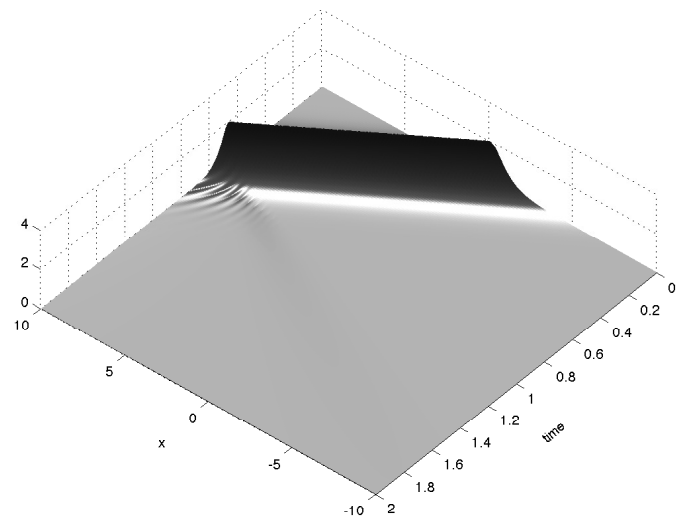

(c) PML

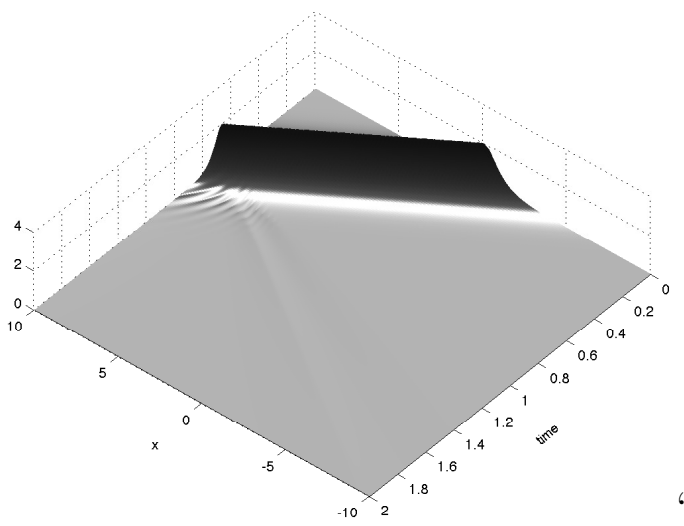

(e) $\mathrm{ABC}$

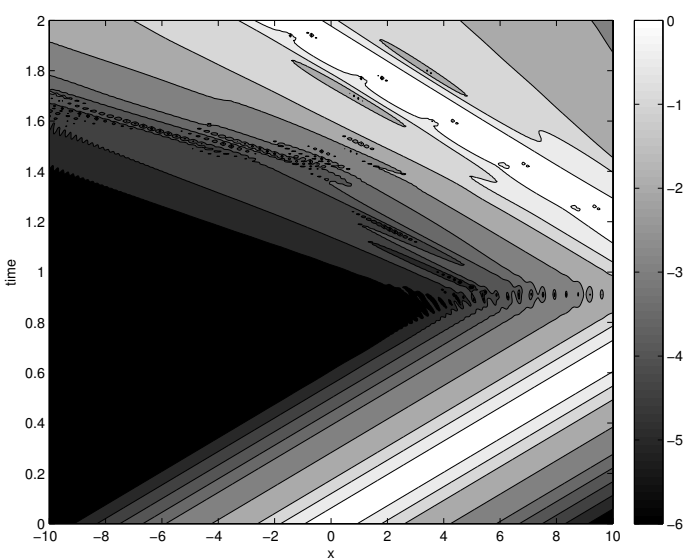

(b) $\mathrm{CAP}$

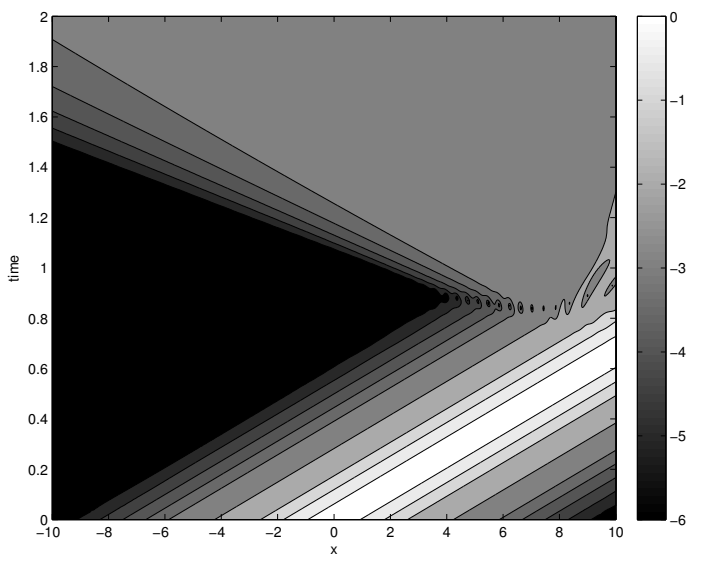

(d) PML

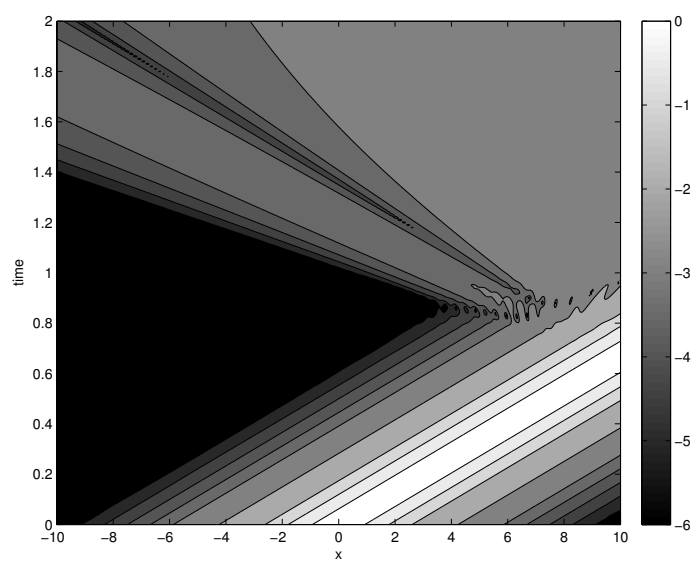

(f) $\mathrm{ABC}$

Figure 3: Numerical solutions $|\psi|$ for the cubic case (left: normal scale, right: log scale). 


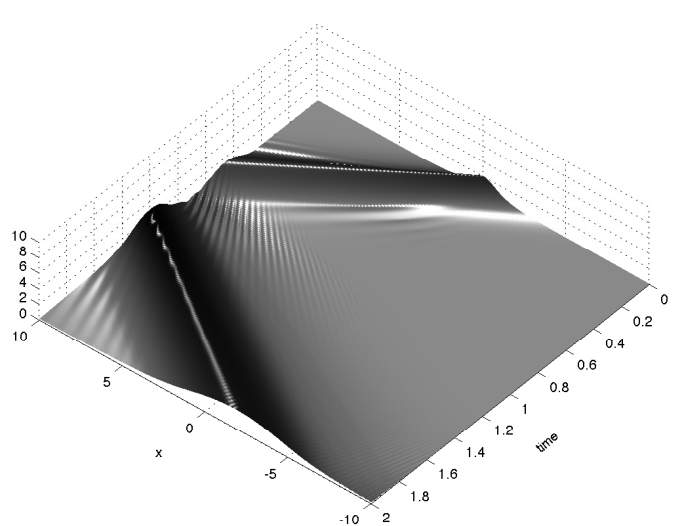

(a) CAP

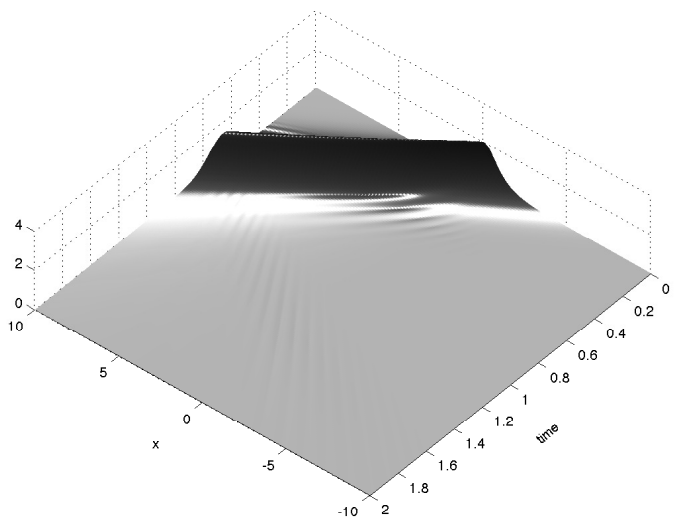

(c) PML

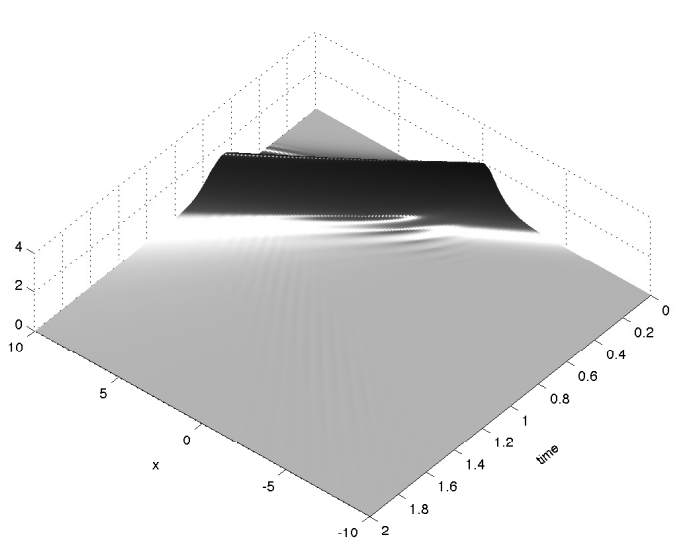

(e) $\mathrm{ABC}$

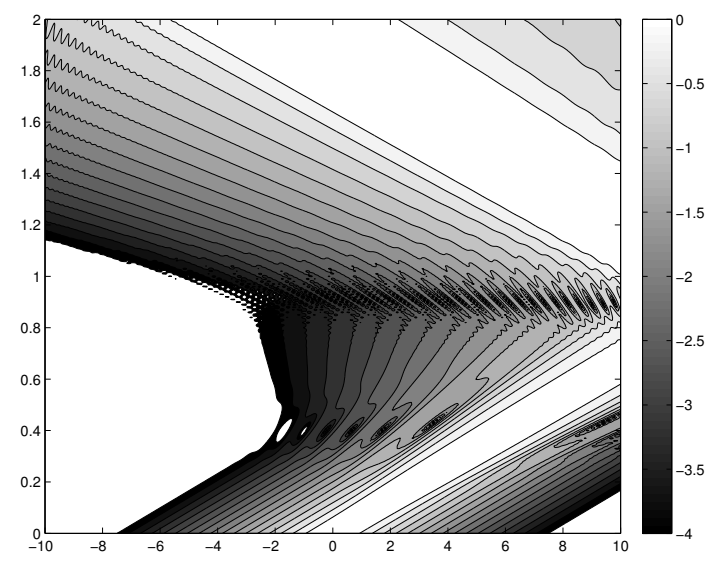

(b) CAP

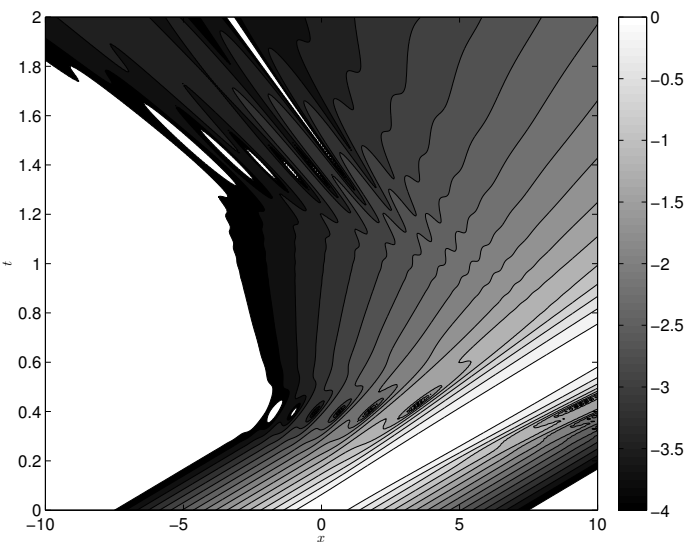

(d) PML

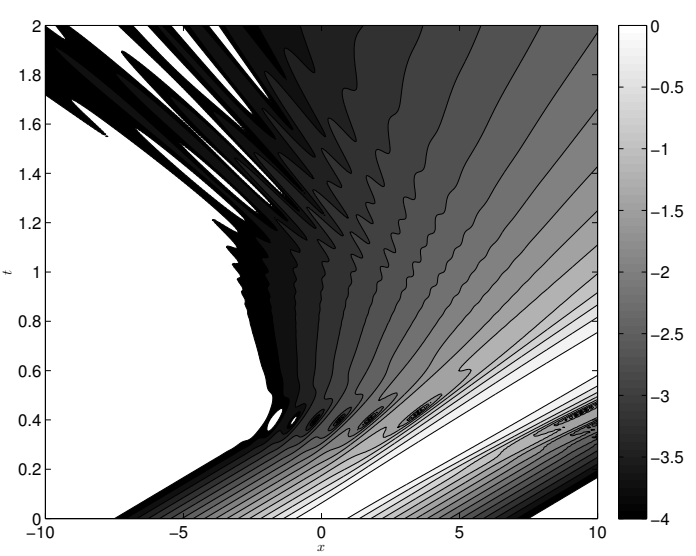

(f) $\mathrm{ABC}$

Figure 4: Numerical solutions $|\psi|$ for the saturation case (log scale). 


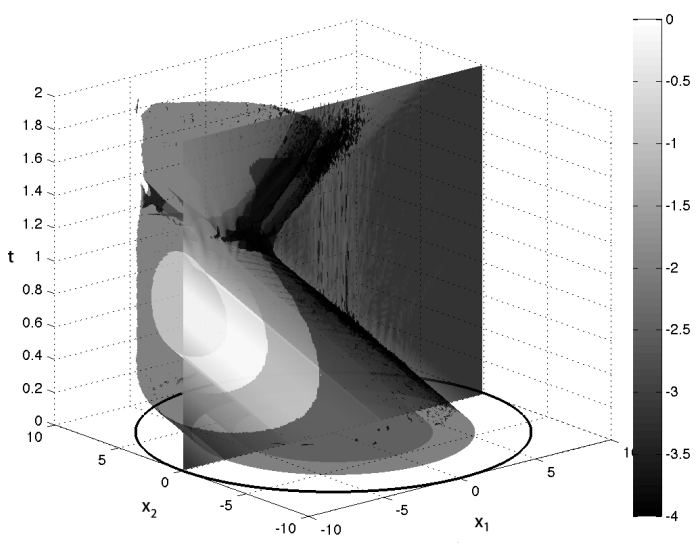

(a) $\mathrm{ABC}$

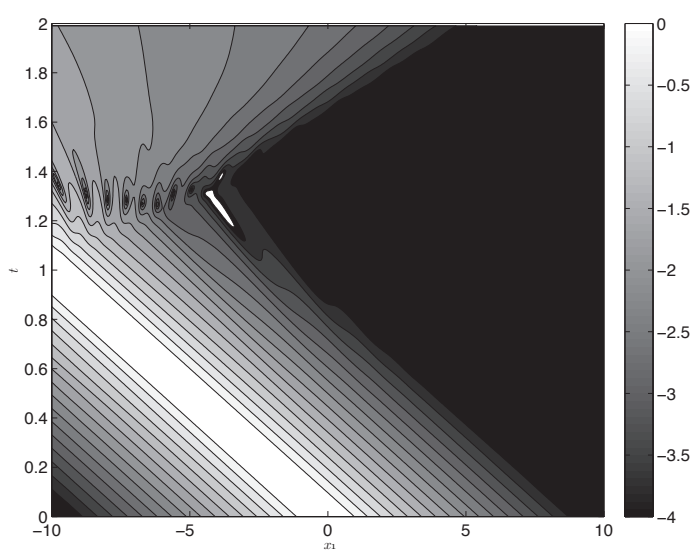

(c) PML

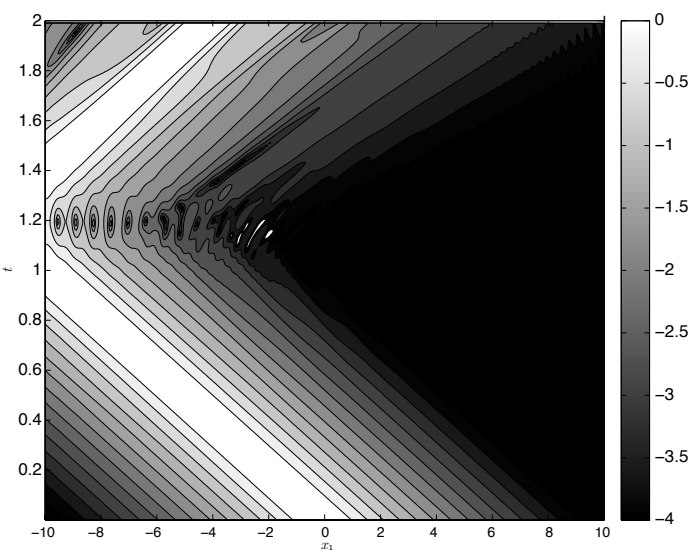

(b) CAP

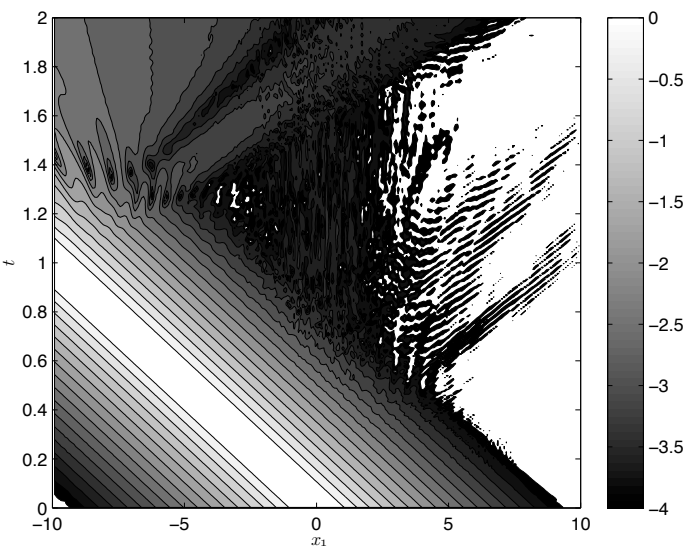

(d) $\mathrm{ABC}$

Figure 5: Numerical solutions $|\psi|$ for the 2D cubic case: Figure 5(a): 3D propagation of the soliton solution on the disk of radius $R=10$ with ABCs. Figures 5(b)-5(d): CAPs, PMLs and ABCs solution. The figures are built as slices in the $\left(x_{1}, t\right)$ plane for $x_{2}=\{0\}$. 